\title{
Lifestyle Behaviors among Nursing Students in Hawler Medical University
}

Kareem Fattah Azeez; Department of Nursing, College of Nursing, Hawler Medical University, Erbil, Iraq. (Correspondence: kareem.fattah@nur.hmu.edu.krd)

\section{ABSTRACT}

Background and objective: The importance of proper nutrition and physical activity in reducing rates of disease and death from chronic diseases has been well established. Poor diet and physical inactivity cause deaths and are major contributors to disabilities that result from diabetes, obesity, and stroke. Drug abuse is a more intense and misuse of drugs can result in addiction. The objectives of this study were to identify lifestyle behaviors among students and to find out association between their lifestyles and some variables (sex, stage, and body mass index) in the College of Nursing in Erbil City.

Methods: A descriptive, cross sectional study was conducted on 100 students from four stages in the College of Nursing (25 students from each stage) during the period December 2016 to April 2017. A questionnaire was composed of two parts, socio-demographic characteristics and questions related to lifestyle behaviors (three domains) among students in the college. Frequency, percentage and chi-square test was used for interpretation of data. Results: Most of the study sample was aged between 18 and 24 years old from urban areas. Near half of them was female and the highest percentage had normal weight based on body mass index assessment tool. There is a non-significant association between lifestyle behaviors and sex among the study sample ( $p$-value $0.312,0.346$ and 0.385 respectively) and with body mass index in activity and nutrition ( $p$-value 0.885 and 0.060 respectively).

Conclusion: Most of the students sometimes practicing the different types of physical activities and exercises, while never abused of substances with sometimes eating more than usual of red meat, salt, and fatty foods. .

Keywords: Lifestyle behaviors; Activity domain; Habit.

$$
\text { Received: } 2 / 9 / 2017
$$

Accepted: $25 / 10 / 2017$

Published: $5 / 5 / 2018$

\section{INTRODUCTION}

The importance of proper nutrition and physical activity in reducing rates of disease and death from chronic diseases has been well established. Poor diet and physical inactivity cause deaths per year and are major contributors to disabilities that result from diabetes, obesity, and stroke. Drug abuse is a more intense and often misuse of drugs may cause addiction. The focus of health promotion and lifestyle are on changing patterns of behavior to promote health rather than simply to avoid illness. Health promotion involves focusing on persons and populations as a whole and diseases. Many factors affect health and these factors are integrated into an individual's lifestyle [1]. Tobacco smoking, diet, and physical exercise are key aspects of lifestyle that influence the risk for the major diseases of rich societies such as cancer and coronary heart disease. Lifestyles and health beliefs appear to be established early in life, setting the pattern for later years [2]. The major determinants of health are socioeconomic factors, lifestyle factors and the physical environment. Lifestyle-related risk factors that were acknowledged in health report of the WHO included unhealthy nutrition, physical 
inactivity, tobacco use and the use of alcohol and illicit drugs [3]. Health-related behavior in early life influences later risks for lifestyle-related disorders. It is therefore important to investigate health behaviors among young people. University students represent a major segment of the young adult population [3]. The promotion of general health and wellbeing [4]. A healthy lifestyle consists of accepting personal responsibility for one's own health, self- fulfillment, health screenings, stress management, healthy diet and regular exercise [5]. Previous studies have suggested that lifestyle choices may account for as much as $50 \%$ of preventable deaths; lifestyle factors during childhood, adolescence, and adulthood are major factors in human disease [6]. Behavior patterns are typically established during adolescence instruction of children and adolescents in health consciousness is more effective than alteration of adult behavior patterns. The extent to which, living conditions, and harmful occupational environments affect the establishment of a healthy lifestyle remains unknown [7]. The objective of the study was to identify lifestyle behaviors among students and identify association between their lifestyles and some variables (sex and of the students) in the College of Nursing in Erbil City.

\section{METHODS}

A descriptive cross sectional study was conducted on 100 students in different years at the College of Nursing/Hawler Medical University from 25 December 2016 to 1 April 2017, in Erbil city. Data were collected through direct interviews with students. A questionnaire format was used for data collection which composed of two parts: part one included demographic characteristics and part two included questions related to three main categories lifestyle behaviors of the Activities (walking, swim- ming, cycling, and running with regular weekly exercise), Substance abuse (smoking, alcohol drinking, and illegal drugs), and nutrition (red meat, salt, and fatty foods). The students responded to the part two through three response options: never, sometimes, and always.

Regarding ethical consideration the permission was taken from Ethical Committee of the college before beginning the research process. Informed consent was obtained from students and they were free to accept or reject the participation. The data were analyzed using SPSS Program version 23. Frequency, percentage, chi-square, and Fisher's exact tests were used.

\section{RESULTS}

Table 1 shows the demographic characteristics of the study participants. Most of the study sample was aged between 18 and 24 years old (95\%), were from urban areas (64\%). Nearly half of them were female $(51 \%)$ and the majority were of normal weight $(72 \%)$, while only $23 \%$ of the participants were overweight and obese based on body mass index assessment tool.

Regarding the life style behaviors of study sample, which are presented in Table 2, the researcher assessed the three main domains of activity, substance abuse, and nutrition. Most of the study sample sometimes practicing the different types of physical activities and exercises like walking, swimming, cycling, and running with regular weekly exercise $(80 \%)$ while most had never abused of substances such as cigarettes, alcohol, and illegal drugs (98\%). More than half of the study sample indicated that they sometimes ate more than a usual amount of red meat, salt, and fatty foods (62\%). 
Table 1: Demographic characteristics of the study sample

\begin{tabular}{lc}
\hline Demographic characteristics & F (\%) \\
\hline Age group (years) & \\
$18-24$ & $95(95)$ \\
$25-31$ & $5(5)$ \\
Living location & \\
$\quad$ urban & $64(64)$ \\
$\quad$ rural & $17(17)$ \\
suburban & $19(19)$ \\
Sex $\quad$ & \\
$\quad$ male & $49(49)$ \\
$\quad$ female & $51(51)$ \\
Body Mass Index $\left(\mathrm{kg} / \mathrm{m}^{2}\right)$ & \\
$\quad$ underweight $<18.5$ & $5(5)$ \\
$\quad$ normal weight $18.5-24.9$ & $72(72)$ \\
$\quad$ overweight $25-29.9$ & $17(17)$ \\
$\quad$ obesity $\geq 30$ & $6(6)$ \\
\hline
\end{tabular}

Table 2: lifestyle behaviors (three domains) female, while most of them had normal BMI. These results agreed with results of the study done by Sauter who found that most of study sample had normal BMI [8]. Majority of the students practiced physical activities so this was agreed with results findings of Spear and Kulbok, which found that majority of study sample practice healthy lifestyles [7]. Regarding the domain of drug abuse, most of the students reported never practicing this habit, which agreed with results of the study of Mamand and Al- Dosky who found that the number of drug abusers in Kurdistan region were less than other country, and that this was related to cultures, social factors and other traditional habits in the Kurdistan Region. Culture is one factor may affect the habit or behaviors [9]. Regarding nutrition domain most of the students responded by sometimes that means majority of them practiced nutrition habit not properly and these findings agreed

\begin{tabular}{lcccc}
\hline \multirow{2}{*}{ Lifestyle behaviors } & Never & Sometimes & Always & Total \\
\cline { 2 - 5 } & F (\%) & F (\%) & F (\%) & F (\%) \\
\hline Activity Domain & $19(19)$ & $80(80)$ & $1(1)$ & $100(100)$ \\
Abuse Domain & $98(98)$ & $1(1)$ & $1(1)$ & $100(100)$ \\
Nutrition Domain & $13(13)$ & $62(62)$ & $25(25)$ & $100(100)$ \\
\hline
\end{tabular}

Table 3 presented the non-significant association between lifestyle behaviors and sex among the study sample ( $p$-value 0.312 , 0.346 and 0.385 respectively). In association between body mass index and lifestyle behavior domains in Table 4, there is only substance abuse has a significant association with body mass index (BMI) ( $p$-value 0.013 ) while activity and nutrition had a non-significant association ( $p$-value 0.885 and 0.060 respectively).

\section{DISCUSSION}

The study revealed that the majority of the cohort were from a city center and were with the study of Bryan et al who found that college students not practiced proper habit regarding nutrition domain [10]. The other results of the study indicate that there was no significant association between sex and three domains of lifestyles, and this finding disagreed with the study of Li et al, which revealed that there was significant association between sex and lifestyles behaviors [11]. The findings of the study indicate that there was a significant association between student's year in school and activity domain while there was significant association between $\mathrm{BMI}$ and abuse domain so this finding was 
Table 3: Association between Lifestyle behaviors and sex

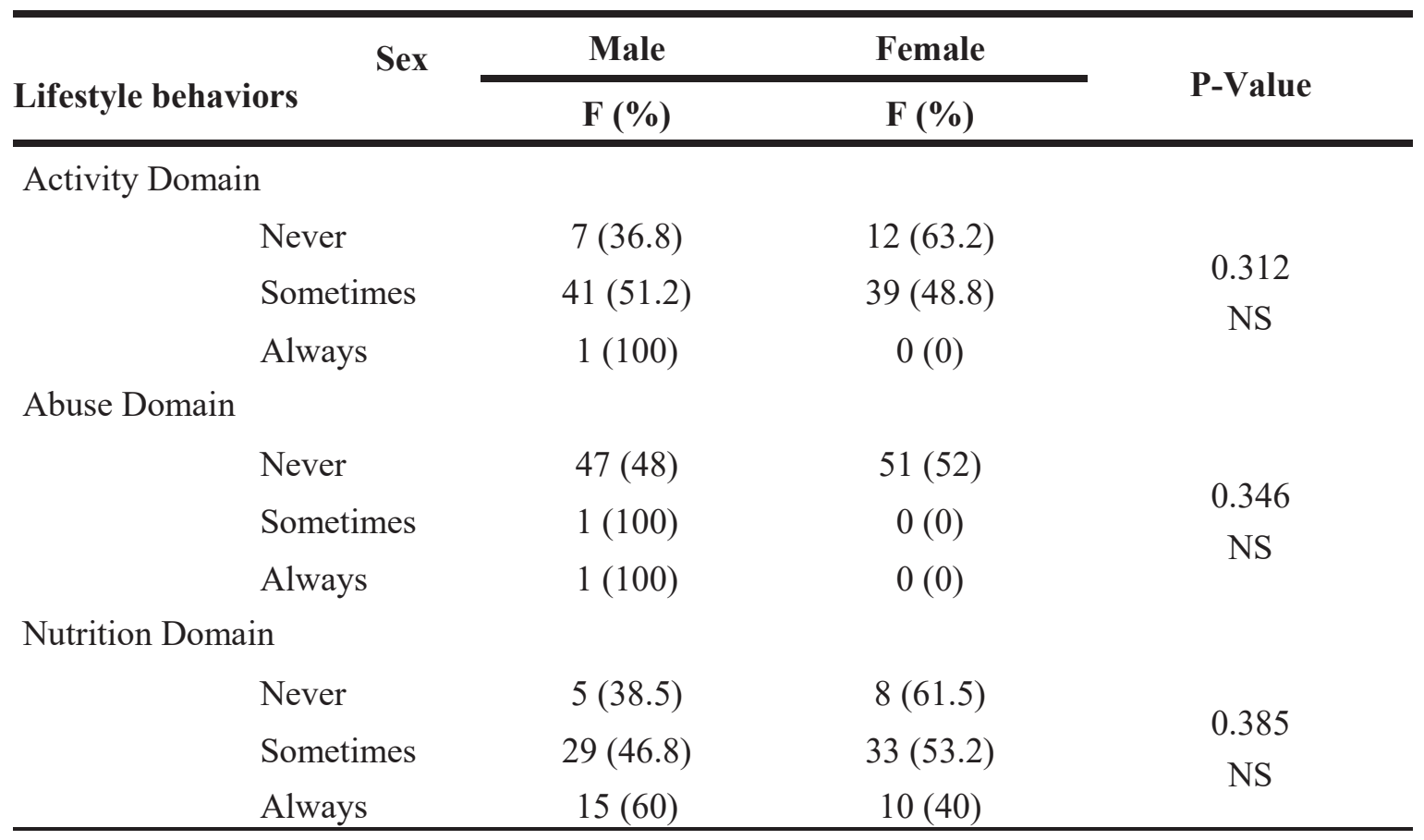

Table 4: Association between BMI and lifestyle behaviors

\begin{tabular}{cccccc}
\hline \multirow{2}{*}{ Life style behaviors } & Underweight & Normal & Overweight & Obesity & \multirow{2}{*}{ P-Value } \\
\cline { 2 - 4 } & $\mathbf{F ~ ( \% )}$ & $\mathbf{F ~ ( \% )}$ & $\mathbf{F ~ ( \% )}$ & F (\%) & \\
\hline Activity Domain & & & & & \\
Never & $0(0)$ & $13(68.4)$ & $4(21.1)$ & $2(10.5)$ & 0.855 \\
Sometimes & $5(6.3)$ & $58(72.5)$ & $13(16.3)$ & $4(5)$ & NS \\
Always & $0(0)$ & $1(100)$ & $0(0)$ & $0(0)$ & \\
Abuse Domain & & & & & \\
Never & $5(5.1)$ & $71(72.4)$ & $17(17.3)$ & $5(5.1)$ & 0.013 \\
Sometimes & $0(0)$ & $1(100)$ & $0(0)$ & $0(0)$ & S \\
Always & $0(0)$ & $1(100)$ & $0(0)$ & $0(0)$ & \\
Nutrition Domain & & & & & \\
Never & $0(0)$ & $12(92.3)$ & $1(7.7)$ & $0(0)$ & 0.060 \\
Sometimes & $2(3.2)$ & $45(72.6)$ & $13(21)$ & $2(3.2)$ & NS \\
Always & $3(12)$ & $15(60)$ & $3(12)$ & $4(16)$ & \\
\hline
\end{tabular}

agreed with study of Barry and Petry who revealed that there was a significant association between BMI and abuse domain [12].

\section{CONCLUSSION}

The majority of students reported sometimes practicing physical activities and exercises, they never abused substances, and sometimes reported eating more than usual amount of red meat, salt, and fatty foods.

\section{CONFLICTS OF INTEREST}

The author reports no conflicts of interest. 


\section{ACKNOWLEDGEMENT}

The author would like to thank all participants and all staff and students who participated in conducting the study.

\section{REFERENCES}

[1] Smith C, Maurer F. Community health nursing; theory and practice. 2 nd ed. W.B. Saunders Company: Philadelphia; 2010.

[2] Steptoe A, Wardle J, Cui W, Bellisle F, Zotti AM, Baranyai $R$ et al. Trends in smoking, diet, physical exercise, and attitudes toward health in European university students from 13 countries. 2010; 35 (2): 97-104.

[3] The European health report 2010. Copenhagen, WHO regional office for Europe, 2010 (European Series, No. 97).

[4] Paredes-López O, Cervantes-Ceja ML, Vigna-Pérez M, Hernández-Pérez T. Berries: improving human health and healthy aging, and promoting quality life-a review. Plant foods for human nutrition. 2010 Sep 1;65 (3):299-308.

[5] Buranatrevedh S, Sweatsriskul P. Model development for health promotion and control of agricultural occupational health hazards and accidents in Pathumthani, Thailand. Industrial health. 2005;43(4):669-76.

[6]Fish C. Health promotion needs of students in a college environment. Public Health Nursing. 1996 Apr 1;13 (2):104-11.

[7] Spear HJ, Kulbok PA. Adolescent health behaviors and related factors: a review. Public health nursing. 2001 Jan 1;18(2):82-93.

[8] Sauter SL. Integrative approaches to safeguarding the health and safety of workers. Industrial health. 2013;51(6):559-61.

[9] WHO. Regional Office for Europe Fact shit report. Alcohol: why .pay attention to this issue during adolescence 2012. Available from http// www.euro.who.int/en/home.

[10] Bryan S, Parasher R, Cahil T, Zipp PG. Yoga, mindful eating, and weight management. Journal of Nutritional Therapeutics. 2013 Dec 30;2(4):173-81.

[11] Li S, Huang H, Cai Y, Xu G, Huang F, Shen X. Characteristics and determinants of sexual behavior among adolescents of migrant workers in Shangai (China). BMC Public Health. 2009 Jun 19;9(1):195.

[12] Barry D, Petry NM. Associations between body mass index and substance use disorders differ by gender: results from the National Epidemiologic Survey on Alcohol and Related Conditions. Addictive behaviors. $2009 \operatorname{Jan} 31 ; 34(1): 51-60$. 